Japan. J. Genetics Vol. 54, No. 5: 371-378 (1979)

\title{
STUDIES ON INTERSPECIFIC HYBRIDIZATION IN RODENTS, II. HISTOLOGICAL OBSERVATIONS ON THE HYBRID EMBRYOS DEVELOPED BY ARTIFICIAL INSEMINATION BETWEEN NORWAY RATS AND BLACK RATS1)
}

\author{
TOSIHIDE H. YOSIDA AND CHOJI TAYA
}

National Institute of Genetics, Misima 411

Received July 9, 1979

Histological examination of hybrid embryos developed by artificial insemination between female Norway rats (Rattus norvegicus, $2 n=42$ ) and male Asian type black rats (Rattus rattus flavipectus, $2 n=42$ ) was carried out with comparison between the hybrid and the control embryos which developed by artificial insemination between female and male Norway rats. Although the retardation of development of the hybrid embryo was observed, the endoderm and ectoderm were found well differentiated on the 7 th and the 9th days. Until the 10th day after insemination, the hybrid embryos were clearly observed in the decidua although the development markedly delayed. The development of mesoderm was poor in this stage. On the 13th day the embryos appeared to be degenerated completely; only rudimental embryonic cells were observed in the decidua. Lethality of the hybrid embryos seemed to be due to the marked retardation of the development and differentiation.

\section{INTRODUCTION}

Embryonic developments of hybrids Norway rats (Rattus norvegicus) and black rats $(R$. rattus) have been described in our previous paper (Yosida ard Taya 1977). In that study we found that among 20 female Norway rats artificially inseminated with the semen of the black rat 9 rats showed pregnancy. However, offspring were never obtained from the interspecific hybridization. In order to obtain further information on the embryonic death of the interspecific hybrids, the embryos were examined histologically. The present paper deals with the results of these examinations with special regard to the comparison of development between the hybrid and the control embryos.

1) Contribution No. 1274 from the National Institute of Genetics, Japan. Supported by grantin-aid for the scientific research from the Ministry of Education, Science and Culture, of Japan. 


\section{MATERIALS AND METHODS}

The female and male Norway rats from the laboratory stock (Fischer or F-344 strain) were used for control experiments. The female Fischer strain rats were also used for the interspecific hybridization experiments. Male Asian type black rats $(R$. rattus flavipectus) which had the same chromosome number and similar karyotype to the Norway rat were used for the present experiments.

The artificial insemination technique has been described in our previous paper (Yosida and Taya 1977). The pregnant females were dissected on the 7th, 9th, 10th, 11th, and 13th day after insemination (counted as the 1th day when the insemination has been taken). The uteri with embryos were cut out and fixed with Bouin's solution. They were embedded in paraffin, sectioned and then stained with Mayer's haematoxylin and eosin.

\section{RESULTS}

\section{Embryos in control experiments}

Differentiation of ectoplacental cone, ectoderm, endoderm and trophectoderm was observed on the 7th day of insemination (Fig. 1A). On the 9th day, the embryos were differentiated into the embryonic and extra embryonic parts. The former consists of the well differentiated embryonic ectoderm and endoderm. The proamniotic cavity was clearly seen. The ectoplacental cone developed at the site of the extra embryonic part (Fig. 2A). On the 10th day the embryo was further differentiated, and three cavities, the ectoplacental, the exocoelom and the amniotic, were formed through the development of chorion and amnion. Apparent differentiation of the mesoderm was observed. Development of the allantois was seen in the exocoelom (Fig. 3A). On the 11th day marked development of the embryo was apparent; the differentiation of the myelocoel, otic placode, foregut and ventricle was observable in this stage (Fig. 4A).

\section{Hybrid embryos by artificial insemination}

On the 7th day after insemination, development of the hybrid embryos was considerably retarded in comparison with the control embryo (Fig. 1B). As seen in the figure, endoderm and embryonic ectoderm were differentiated, though development of the ectoplacental cone was poor. The retardation of the development of the embryo was seen on the 9th day (Fig. 2B). Although the differentiation into embryonic and extraembryonic parts took place, it was incomplete. Poor differentiation of the embryonic ectoderm, the endoderm, and the ectoplacental cone were recognized, although the proamniotic cavity was formed. On the 10th day the differentiation of the hybrid embryo seemed to be more retarded and they did not have the three cavities, but still possessed the proamniotic cavity. Although the differentiation of the mesoderm was seen in this stage, its differentiation was poor (Fig. 3B). On the 13th day, the hybrid embryo degenerated completely and only rudimental cells were observed in the embryonic sac (Fig. 4B). 


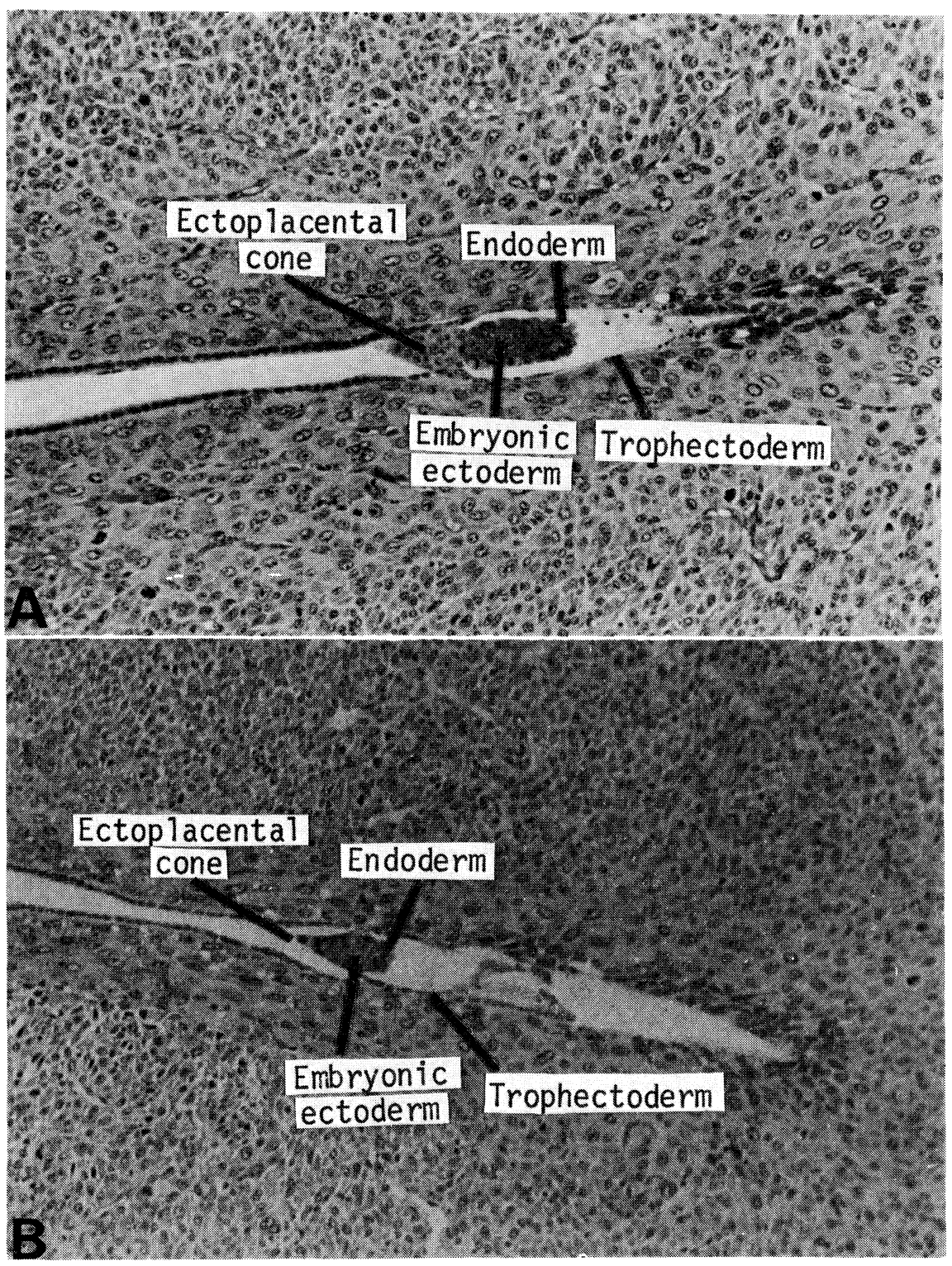

Fig. 1. Longitudinal section of the 7th day embryos. A, control embryo developed by artificial insemination between female and male Norway rats. B, hybrid embryo developed by artificial insemination between the female Norway and male black rats. Differenteation of endoderm and embryonic ectoderm was observed in both embryos, while that of the ectoplacental cone in the hybrid is poor in this stage. 


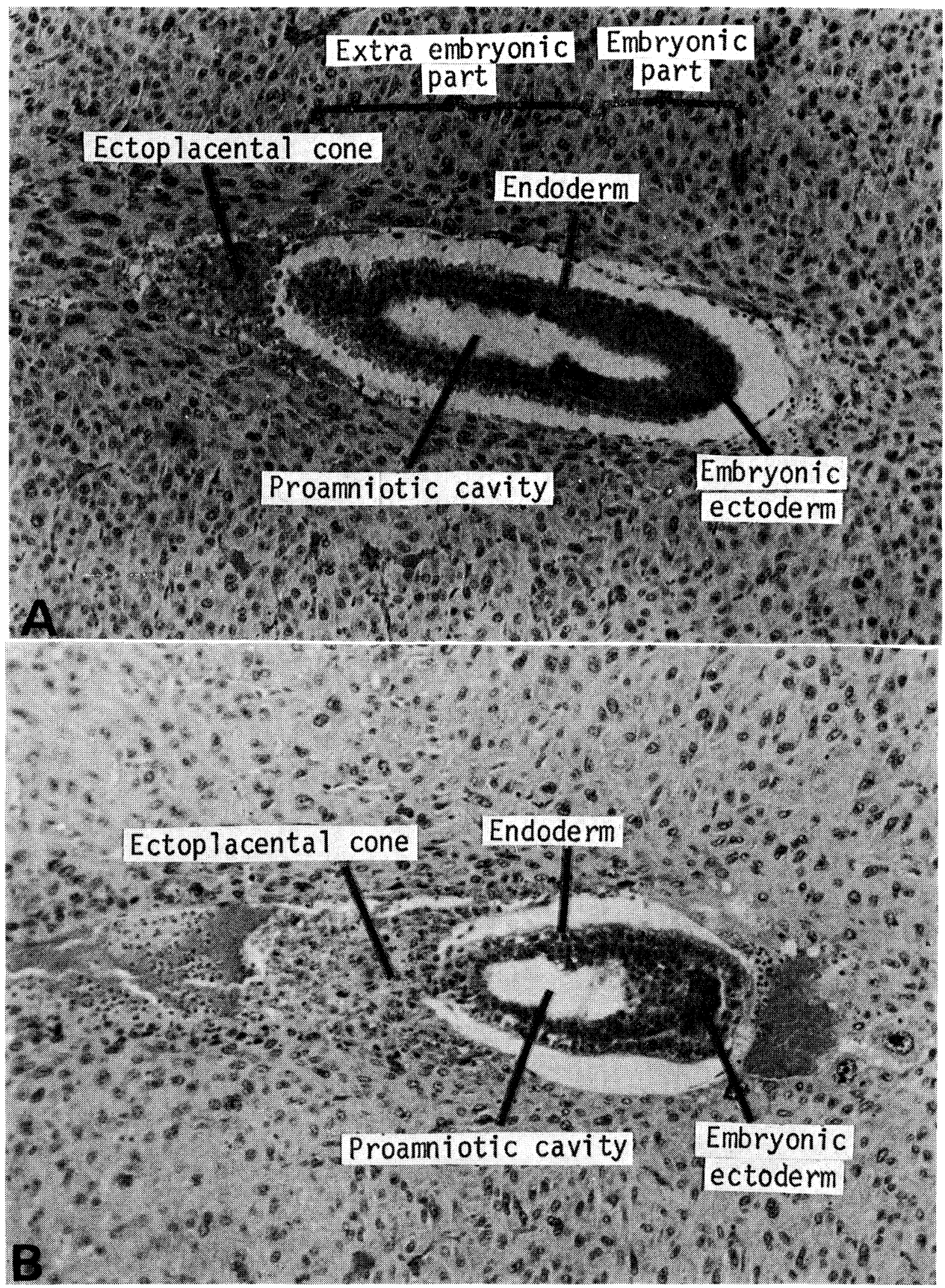

Fig. 2. Longitudinal section of the 9th day embryos. A, control embryo. Tine embryo is differentiated into embryonic and extra embryonic parts. Development of embryonic ectoderm is notable. B, hybrid embryo. The differentiation into embryonic and extra embryonic parts is barely seen. Development of embryonic ectoderm is poor. Ectoplacental cone is developed as well as in control. 


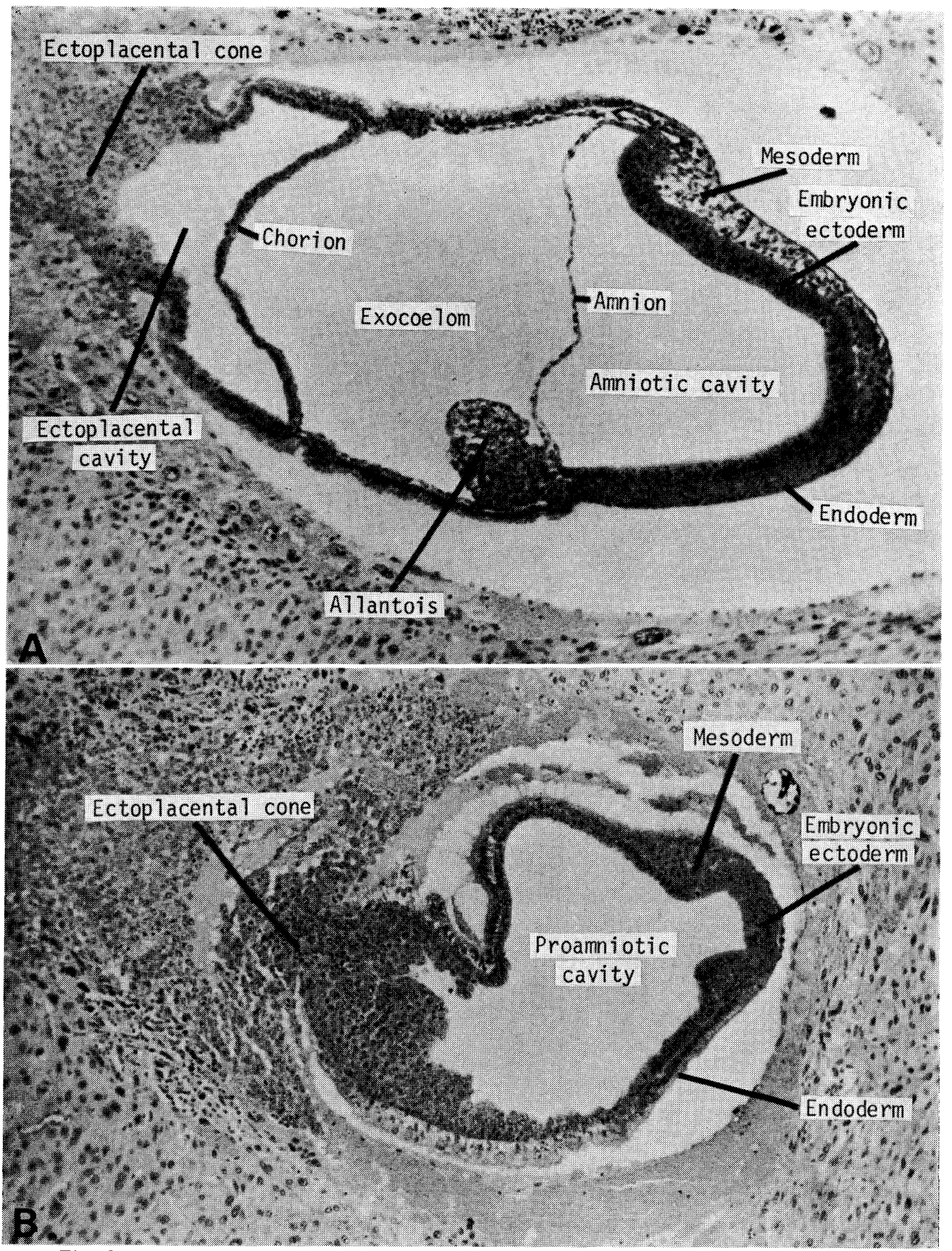

Fig. 3. Longitudinal section of the 10th day embryos. A, control. Amniotic cavity, exocoelom and exoplacental cavity are well recognized through the development of the amnion and the chorion. Differentiation of the mesoderm and the allantois is clearly seen. B, hybrid embryo. Three cavities seen in the control embryo are not yet formed. Poor differentiation of mesoderm can be seen. Irregular growth of the ectoplacental cone is shown. 


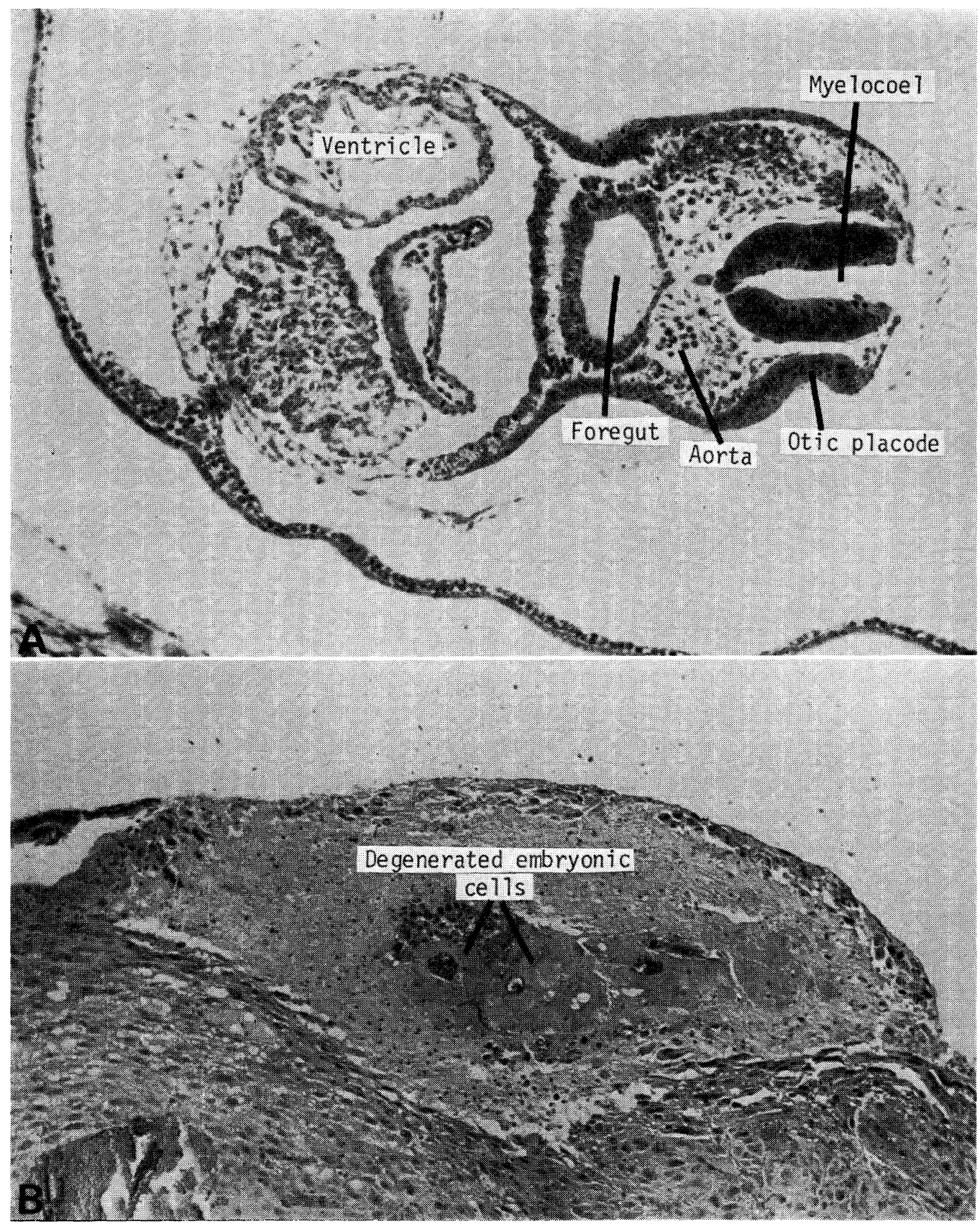

Fig. 4. A, longitudinal section of the 11th day embryo in control experiment. Various well differentiated organs are identified from the figure. $B$, section of the 13th day hybrid embryo. Embryo degenerated completely; only a mass of rudimental cells is recognized. 


\section{DISCUSSION}

Based on the observation of karyotypes in the black rat and the Norway rat, we (Yosida 1973; Yosida and Sagai 1973) have demonstrated that the karyotype of the Norway rat is rather similar to the Asian type black rat, but not to the Oceanian and Ceylonese type black rats, and concluded that the karyotype of the Norway rat could be derived from one of the polymorphic karyotypes in the Asian type black rats. Although these two species are karyotypically similar, the hybrids between them have not been obtained in a natural or by a laboratory mating. In an attempt to obtain the hybrid, artificial insemination between them was taken place. However, no hybrid offspring have been obtained so far (Hiraiwa and Yoshida 1955a, b; Yosida and Taya 1977).

On the present examination, the hybrid embryos between them were well differentiated up to about the 7th day. Nevertheless it was followed by a marked retardation of embryonic development. On the 9 th and 10th days after insemination, a remarkable arrest of the development of embryo was observed, and then the hybrid embryo degenerated as seen in the figure. It is difficult to determine the cause of degeneration of the hybrid embryo from the present histological observation. The retardation of the development of the hybrid embryo could be seen from the histology of all stages examined, although the control embryos have developed normally as seen in natural matings.

On the cause of embryonic death in the interspecific hybridization between the Norway and black rats, two explanations have been considerd by us (Yosida and Taya 1977). One of them is the immunological reaction in the female parent and the hybrid embryo which developed by mating between the different species, and the other due to the developmental disorder of the hybrid itself by genic imbalance. The karyotype of the hybrid embryo has been demonstrated by us to be of a normal diploid, consisting of each haploid set from both parents (Yosida and Taya 1977). Due to the difference of heterochromatic C-bands in these two species (Yosida 1975), they emphasized a possibility of the latter event. Recently Yosida (1978) has demonstrated that the nucleolus organizer (Ag-NORs) of chromosomes were clearly different between these two species. The NORs are considered to be sites of the r-RNA genes important to genetic expression (Goodpasture and Bloom 1975; Miller et al. 1976). These differences seem to be another important factor to the embryonic death of the hybrids, but the further monitor on the structure and the function of the NORs, the heterochromatin and some other aspects in the chromosomes of the hybrid embryo will be necessary to resolve the above problem. An incompatibility between chromosomes of the black rat and the cytoplasm of egg cell in the Norway rat should be considered as one of the genic unbalance in the hybrid embryo. This is also remained as the further problem. 


\section{LITERATURE CITED}

Goodpasture, C., and S. E. Bloom, 1975 Visualization of nucleolar organizer regions in mammalian chromosomes using silver staining. Chromosoma (Berl.) 53: 37-50.

Hiraiwa, Y. K., and H. Yoshida, 1955a Conception by the cross between Rattus norvegicus and $R$. rattus. I. Activation of the corpus luteum and properties of the sperm suspension in the artificial insemination of the albino rat. (Japanese with English resume). Sci. Bull. Fac. Agr. Kyushu Univ. 15: 123-128.

Hiraiwa, Y. K., and H. Yoshida, 1955b Conception by the cross between Rattus norvegicus and R. rattus. II. Breeding experiments by artificial insemination (Japanese with English resume). Sci. Bull. Fac. Agr. Kyushu Univ. 15: 267-273.

Miller, O. J., D. A. Miller, V. G. Dev, R. Tantravahi, and C. M. Croce, 1976 Expression of human and suppression of mouse nucleolus organizer activity in mouse-human somatic cell hybrids. Proc. Natl. Acad. Sci. USA 73: 4531-4535.

Yosida, T. H., 1973 Evolution of karyotypes and differentiation of 13 Rattus species. Chromosoma (Berl.) 40: 285-297.

Yosida, T. H., 1975 Diminution of heterochromatic C-bands in relation to the differentiation of Rattus species. Proc. Jap. Acad. 51: 659-663.

Yosida, T. H., 1978 A preliminary note on silver stained nucleolar organizer regions in the black and Norway rats. Proc. Jap. Acad. 54, Ser. B: 353-358.

Yosida, T. H., and T. Sagai, 1973 Similarity of Giemsa banding patterns of chromosomes in several species of the genus Rattus. Chromosoma (Berl.) 50: 283-300.

Yosida, T. H., and C. Taya, 1977 Studies on interspecific hybridization in the rodents, I. Artificial insemination between the Norway rat (우) and black rat ( $\hat{\delta})$ and the resulting karyotypes in the hybrid blastocyst. Japan, J. Genetics 52: 289-299. 